\title{
LE SYSTÈME MUSCULAIRE DU SCOLEX ECHINOCOCCIQUE
}

\author{
MÉCANismes DE DÉVAGINATION ET D'INVAGINATION
}

DU ROSTRE ET DES VENTOUSES

\section{Par F. Coutelen, J. Biguet, J.-M. Doby et $\boldsymbol{s}$ t. DeBlock}

En 1856, ayant étudié, sur le vivant, la naissance et le développement du scolex échinococcique aux dépens de la capsule proligère de l'hydatide, Leuckart s'étonnait de la grande et évidente contractilité de la future petite tête de Ténia : «Bien qu'elle ne soit formée seulement que de cellules », écrivait-il, * elle s'allonge, elle se contracte, bientôt après, de la moitié de sa longueur antérieure; elle se fléchit et se balance à droite et à gauche comme un pendule et, souvent même, elle se renverse à l'intérieur de la capsule, en sorte que la cuticule la recouvre alors extérieurement, comme cela s'observe chez les petites têtes échinococciques complètement développées 》. La formation précoce de fibres musculaires dans le bourgeon initial avait échappé à Leuckart, qui ne pratiquait pas de coupes histologiques, comme elle devait passer inaperçue, pour la même raison, des anciens helminthologistes qui avaient étudié, de leur côté, ce développement (Wagener, 1854 ; Naunyn, 1862 ; Rasmussen, 1865 ; Moniez, 1880).

C'est Goldschmidt, le premier (1900), qui, reprenant cette étude sur des préparations fixées et colorées, a observé l'apparition de mycblastes et de fibres musculaires dès les premiers stades de développement du scolex échinococcique sur la capsule proligère. Il marque nettement, sur ses dessins (planche XXXIII, figures de 1 à 11), la coupe transversale punctiforme des fibres circulaires souscuticulaires, mais laisse plutôt deviner, quoiqu'il en parle assez longuement dans son texte, l'existence d'autres fibres moins nombreuses : longitudinales et sous-cuticulaires, transversales ou circulaires au niveau du rostre, et même « dorso-ventrales» (1) au

(1) Cette appellation nous paraît impropre, et en tout cas prèmaturée, lorsqu'il s'agit de scolex échinococciques en voie de formation, dont toute l'organisation s'effectue symétriquement autour d'un axe antéro-postérieur et auxquels on ne saurait distinguer une face dorsale et une face ventrale.

Ann. de Parasitologie, T. XXVII, N 1-2-3. - 1952. 
niveau du corps du scolex. Les noyaux des cellules myoblastiques du rostre et des ventouses, mais non leurs fibres musculaires, sont, tout au contraire, très apparents sur trois de ses dessins, et, en particulier sur sa figure 6.

Après cette date et à notre connaissance, les très nombreux auteurs qui ont étudié et dessiné des scolex échinococciques, mème d'après des coupes histologiques, n'ont pas rappelé, dans leurs publications, les résultats de ce premier travail de Goldschmidt. Il marque cependant, au stade embryonnaire, l'apparition d'un système musculaire; et, si l'on excepte la figuration traditionnelle d'une musculature au niveau du rostre et des ventouses, sous forme schématique de traits plus ou moins parallèles irradiés de la profondeur à la superficie de ces crganes, il ne semble pas y avoir eu, par la suite, un travail d'ensemble sur la question.

C'est un essai de ce genre que nous avons voulu tenter, en pensant qu'il pourrait prendre place dans une ètude comparative des descriptions histologiques qui ont été faites antérieurement pour quelques autres formes larvaires de Téniidés. D'autre part, la présentation topographique d'un tel système musculaire nous a paru également utile pour comprendre les mouvements si nombreux et si complexes du scolex et, plus particulièrement, pour analyser les phénomènes de dévagination et d'invagination que présente sa partie antérieure.

Nous avons donc étudié ce système musculaire du scolex échinococcique et sa physiologie, d'une part à frais, sur des scolex adultes, normaux ou arrivés à des stades de vésiculation régressive plus ou moins avancée ; d'autre part, nous nous sommes servis de préparations, fixées ou colorées par des techniques diverses. L'un de nous, décrivant antérieurement le système excréteur des Hydatides échinococciques, a montré les avantages que l'on peut tirer de l'observation de scolex ayant subi in vitro un début d'évolution vésiculaire, qui dissocie opportunément les divers éléments histologiques de la petite tête et en facilite beaucoup l'examen.

Cet ensemble musculaire est très compliqué. Pour en rendre plus aisée la description topographique, on peut, schématiquement, le diviser en quatre groupes, histologiquement et physiologiquement distinets :

1) Un groupe musculaire sous-cuticulaire et dévaginateur.

2) Un groupe musculaire fonctionnel et mobilisateur des ventouses.

3) Un groupe musculaire fonctionnel du rostre et des crochets.

4) Un groupe musculaire profond, invaginateur. 
Il existe, en dehors de ces quatre grands groupes, un certain nombre de fibres musculaires, isolées ou non, dont il nous a été impossible de préciser les insertions, dont le trajet est difficile à suivre même sur le frais ou sur coupes sériées, mais qui pourraient, sans doute, être rattachées à l'un ou l'autre de ces systèmes par leur action physiologique. La densité et le tassement des divers éléments histologiques que réunit, sous un aussi faible volume, un scolex échinococcique microscopique, en rendent la lecture souvent malaisée, et ce n'est guère que sur des scolex dévaginés et légèrement vésiculeux, que de telles observations sont possibles. C'est, par contre, un matériel de choix pour diverses études histo-physiologiques sur les cestodes.

\section{Groupe musculaire sous-cuticulaire et dévaginateur}

(Planche I, figures 1 et quadrant 1 de la figure 4)

Il constitue, superficiellement et dans la zone sous-cuticulaire, un réseau extrêmement serré, composé de deux couches superposées de fibres musculaires, l'une comprenant des fibres circulaires externes exactement appliquées à la face interne de la cuticule, l'autre, sous-jacente à la première, composée de fibres longitudinales perpendiculaires aux précédentes. Là où elles coexistent, ces deux couches de fibres musculaires forment un quadrillage caractéristique bien visible, optiquement, sur le frais (microphoto $n^{\circ} 5$ ). Ce quadrillage s'étend, à la partie postérieure du scolex, jusque sur son pédicule (planche I, figure 2, dans le bas), et certaines fibres sont même décelables, à l'entcur de l'insertion du pédicule, sur la capsule proligère elle-même. Ce sont peut-être quelques-unes de ces fibres sous-cuticulaires qui furent signalées par Wagener, en 1854, sur de petites hydatites étudiées à frais ; il les avait toutefois localisées non sur la capsule, mais sur la membrane proligère, où il n'est pas impossible, du reste, qu'il puisse s'en former exceptionnellement. Nous avons dit, au début, que Goldschmidt (1900) a marqué, sur ses dessins, la section transversale de fibres musculaires apparues très tôt sous la fine pellicule cuticulaire interne de la capsule proligère, lors même de la formation de l'épaississement de son parenchyme; le jeune bourgeon, qui donnera ultérieurement naissance à un scolex échinococcique, différencie donc déjà des myoblastes et des fibres musculaires.

a) Les fibres circulaires externes de la sous-cuticule s'étagent de la partie postérieure du scolex, pédicule compris, jusqu'en avant et au voisinage de la partie la plus antérieure du rostre (microphoto 
$n^{\circ} 6$ ). Toutefois, une large bande circulaire de la sous-cuticule, limitée en avant par le bord antérieur des ventouses et, en arrière, par leur bord postérieur, parait en être dépourvue (planche I, fig. 1, et microphoto 11). La section punctiforme de ces fibres circulaires, surtout à la partie postérieure du scolex, est bien visible sur des coupes histologiques (microphoto $\mathrm{n}^{\circ} 12$ ).

b) Les fibres longitudinales internes de la sous-cuticule, immédiatement sous-jacentes aux précédentes, s'étendent de la partie postérieure du scolex, pédicule compris, au bord postérieur saillant des ventouses; elles dépassent donc légèrement le « collet » souvent rétréci qui délimite en deux régions, antérieures et postérieures, le corps du scolex. Quatre larges faisceaux de ces fibres longitudinales se prolongent toutefois dans la partie antérieure du scolex, entre les quatre ventouses, pour aller s'insérer bien en avant d'elles à la base du rostre, au-dessous de la double couronne de crochets (planche I, figure 1).

c) La contraction des fibres circulaires externes de la zone souscuticulaire tend vraisemblablement à allonger le scolex dans le sens antéro-postérieur; la contraction des fibres longitudinales tend à le raccourcir et à augmenter son diamètre transverse; leur jeu combiné explique les ondes de contraction que l'on peut observer à frais dans des sens divers.

Enfin et surtout, la contraction synergique et puissante du quadrillage que forment ces fibres musculaires circulaires et longitudinales situées dans la partie postérieure du corps du scolex, permet d'expliquer le phénomène de la dévagination. Ces fibres, en effet, en se contractant, compriment le contenu de cette partie postérieure, tendant à l'expulser ; c'est en fait le rostre et les ventouses, de la partie antérieure de la petite tête, repliée en doigt de gant à l'intérieur de la partie postérieure durant l'invagination, qui sont projetés en avant et à l'extérieur. On peut aussi admettre que les bandes de fibres musculaires longitudinales, qui passent entre les quatre ventouses et s'insèrent d'une part à la base du rostre en avant et, d'autre part, en arrière, à la partie postérieure du scolex, peuvent jouer un rôle accessoire dans cette dévagination.

\section{Groupe musculaire fonctionnel et mobilisateur des ventouses}

(Planche I, figures 1, 2, 3 et moitié 2 de la figure 4)

Ce système musculaire, très compliqué, comprend à la fois des fibres musculaires superficielles et d'autres fibres plus ou moins 
profondes ; les unes, puissantes, servent à la contraction fonctionnelle de cet organe de fixation; les autres assurent soit les déplacements individuels de chaque ventouse dans des directions diverses, soit des déplacements conjugués de deux ou plusieurs ventouses à la fois. Quant aux faisceaux musculaires profonds, enfin, ils les tirent en dedans et vers l'arrière et concourent par conséquent à l'invagination globale de la partie antérieure du scolex dans sa partie postérieure, comme nous le verrons plus bas.

a) Musculature superficielle des ventouses. - Elle est logée dans la zone sous-cuticulaire (planche I, figure 1). Elle comprend :

- Des fibres circulaires contenues dans le bord saillant externe de la ventouse. Leur contraction réduit la surface d'application de l'organe.

- Un réseau quadrillé de fibres transversales et longitudinales situées dans le fond de la ventouse, sous sa mince cuticule (microphoto $n^{\circ} 7$ ). Leur contraction, suivant le cas, ou bien allonge la ventouse dans le sens entéro-postérieur ou bien l'élargit dans le sens transversal. Leur contraction simultanée tend à diminuer la surface du fond de l'organe.

- Un faisceau de fibres musculaires insérées sur chaque bord latéral de la ventouse, dirigé en arrière vers la ligne médiane et s'entrecroisant à son niveau, avec le faisceau homologue issu de la ventouse voisine. La contraction d'un tel faisceau tire le bord de la ventouse en arrière et par côté ; les deux faisceaux se contractant ensemble, la tirent en arrière, vers le collet.

b) Musculature fonctionnelle du corps de la ventouse (planche I, figures 2,3 et 4 , microphoto $n^{\circ} 11$ ).

Située plus profondément que la précédente (planche I, figure 3 et microphoto $\mathrm{n}^{\circ} 11$ ), cette musculature comprend d'abord un réseau de fibres radiées, puissantes, insérées à peu près perpendiculairement d'une part sur la face cuticulaire externe de l'organe, et, d'autre part, sur la base interne et profonde de la ventouse. On distingue nettement, sur la microphoto $\mathrm{n}^{\circ} 11$, ces fibres et leurs myoblastes ; elles sont inscrites par ailleurs, sous un aspect radié, sur la figure 3 de la planche I. C'est là le système musculaire fonctionnel de l'organe ; il est toujours schématiquement représenté sur les figures des auteurs. Sa contraction tend à faire le vide dans la cupule de la ventouse, après qu'elle a appliqué son bord sur un support et qu'elle a immobilisé sa face basale profonde comme un point d'insertion fixe. Les fibres profondes rétractrices doivent aider à cette immobilisation. 
Il existe aussi des fibres circulaires plus internes (planche I, figure 2), dont la contraction tend tout à la fois à rétrécir la surface de la cupule, à l'allonger dans son grand axe et à en faire saillir les bords à l'extérieur.

c) Musculature profonde assurant la mobilisation du corps des ventouses (planche I, figures 2,3 et 4 , microphotos $\mathrm{n}^{\circ s} 8,9$ et 10).

Cet ensemble musculaire, inséré sur les faces latérales du corps de la ventouse, comprend, en allant de l'extérieur vers la profondeur du scolex :

Six à huit grosses fibres musculaires insérées sur la face latérale de la ventouse, dirigées sur le côté en avant, s'entrecroisant sur la ligne médiane, en un chiasma bien visible à frais (microphotos $n^{\circ s} 8$ et 9 ), avec celles de la ventouse adjacente. Ces fibres passent obliquement en avant de la face antérieure de cette ventouse ; elles diminuent de volume au fur et à mesure qu'elles se rapprochent les unes des autres. Il nous a été impossible de distinguer si elles allaient se fixer sur la cuticule de la face antérieure du scolex, au niveau de la ligne médiane séparant deux ventouses, ou bien si elles s'anastomosaient avec les fibres homologues venues de la face opposée et provenant de la ventouse située symétriquement par rapport à l'axe antéro-postérieur du scolex. Dans le premier cas, ces muscles tirent en-dedans et en avant la ventouse qu'ils mobilisent ; dans le second cas, leurs contractions synergiques tirent dans le même sens les deux ventouses symétriques situées sur les deux faces opposées.

- Plus profondément encore, et en arrière, se trouve un autre groupe de fibres plus fines s'insérant également sur la face latérale interne de la ventouse ; elles croisent sur la ligne médiane, en un second chiasma, le faisceau homologue de la ventouse voisine, et se dirigent vers le côté en arrière (planche I, figure 2). Le faisceau contourne la face postérieure de cette ventouse adjacente (microphoto $\mathrm{n}^{\circ} 10$ ), et, là encore, nous ne pouvons préciser s'il s'insère jmmédiatement sur la face interne de la cuticule du scolex, ou s'il s'anastomose avec le faisceau homologue de la ventouse située sur la face opposée, symétriquement par rapport à l'axe antéro-postérieur. La contraction de ce faisceau de fibres musculaires tire la ventouse sur le côté et en arrière dans le premier cas ; dans le second cas ce sont les deux ventouses symétriques par rapport à l'axe qui sont ensemble tirées dans la même direction.

- Enfin, quelques fibres musculaires unissent deux à deux les ventouses sur une même face ; les unes, insérées sur les faces laté- 
rales, s'entrecroisent sur la ligne médiane; d'autres, de direction parallèle, s'insèrent sur la base même des ventouses (planche 1, figure 3). Leur contraction rapproche transversalement les deux ventouses voisines.

\section{Groupe musculaire fonctionnel du rostre et des crochets}

(Planche I, figures 3 et 4 , microphoto $\mathrm{n}^{\text {? 11) }}$

Dans sa partie superficielle la plus antérieure et la plus proéminente, en avant des crochets, le rostre présente quelques fibres musculaires circulaires ou entrecroisées en sens divers (planche I, figure 2). Il est possible que, plus profondément, il existe aussi des fibres circulaires à la base du rostre, mais il nous a été impossible de les déceler avec certitude.

Au-dessous de cette partie antérieure du rostre, maintenant totalement close en avant, après avoir donné naissance aux crochets durant le développement du scolex, se trouve le «coussin » rostral : c'est une formation musculaire rappelant identiquement celle qui caractérise le corps d'une ventouse. Elle est formée également de fibres musculaires puissantes, orientées d'avant en arrière, légèrement radiées, s'insérant sur les deux faces antérieures et postérieures du 《coussin (microphoto $\mathrm{n}^{\circ} 11$ ). Elles constituent la musculature fonctionnelle du rostre ; c'est leur contraction qui, attirant d'avant en arrière la face antérieure du « coussin », fait basculer en même temps et dans la même direction l'ensemble des manches des crochets qui lui sont solidaires; leurs lames libres sont alors projetées en avant, cependant que leurs pointes s'écartent en même temps l'une de l'autre et du pourtour du rostre. Ce sont ces fibres musculaires du rostre, déjà bien connues elles aussi, qui sont toujours figurées dans les représentations schématiques des auteurs, avec celles des ventouses, sur des scolex de cestodes adultes ou larvaires, et comme nous l'avons rappelé antérieurement. On peut se rallier à l'opinion des helminthologistes, qui depuis Leuckart (1856) homologuent le rostre à une ventouse, tant la structure histologique de ces deux organes est identique. Seul le rôle fonctionnel a changé.

\section{Groupe musculaire profond, invaginateur}

(Planches I, figures 3 et 4 , microphoto $\mathrm{n}^{\circ} 12$ )

Ce système musculaire profond, invaginateur dans son ensemble, est formé de muscles rétracteurs et invaginateurs des quatre ventouses et de muscles rétracteurs et invaginateurs du rostre. 
a) Muscles invaginateurs des ventouses. - Ils constituent, pour chaque ventouse, deux groupes de fibres msculaires, toutes insérées, en avant, sur la face profonde et basale de la ventouse.

Un faisceau se dirige d'avant en arrière et va s'insérer d'autre part latéralement sur la face interne de la cuticule des scolex, dans sa partie postérieure. Un autre faisceau, plus profond, se dirige en arrière et en dedans, pour aller s'insérer sur le pédicule, à côté et autour de l'insertion, plus interne et axiale, du faisceau rétracteur du rostre (planche I, figure 3 et quadrants de la figure 4).

b) Muscles invaginateurs du rostre. - lls constituent un faisceau puissant de fibres musculaires nombreuses et serrées, dirigées suivant l'axe antéro-postérieur du scolex. Ces fibres s'insèrent, en avant, sur la face postérieure de la membrane basale du « coussin » musculaire rostral, et, en arrière, sur la face postérieure du scolex, au niveau du pédicule (planche I, figure 3 et microphoto $\mathrm{n}^{\circ}{ }^{\circ} 12$ ). La contraction isolée de ce faisceau musculaire attire le rostre en arrière et le rétracte. Par ailleurs, on conçoit que son action synergique avec celle des groupes de fibres musculaires rétracteurs des ventouses entraîne toute la partie antérieure du scolex à l'intérieur de la partie postérieure et détermine ainsi l'invagination complète de la petite tête.

\section{RÉsuné}

Nous décrivons, dans ce travail, le système musculaire du scolex échinococcique. Il a été étudié à la fois sur le vivant, avec un éclairage approprié, et sur des coupes histologiques. Les scolex ont été examinés soit à l'état de complet développement, soit à des stades divers d'évolution vésiculaire régressive.

. Ce système musculaire, compliqué, est formé de très nombreuses fibres dont il est souvent malaisé de distinguer les myoblastes, tassés parmi les cellules du parenchyme, les cellules à corpuscules calcaires, les cellules excrétrices à flamme vibratile, et les cellules canaliculaires. Seules les cellules myoblastiques du coussin du rostre et des ventouses sont nettement perceptibles. On peut néanmoins suivre les fibres musculaires elles-mêmes, à frais, et en donner une systématisation d'ensemble. Au point de vue histophysiologique, il y a lieu d'envisager ainsi quatre groupes fonctionnels :

- un groupe musculaire sous-cuticulaire et dévaginateur ;

- un groupe musculaire fonctionnel et mobilisateur des ventouses ; 
- un groupe musculaire fonctionnel du rostre el des crochets ;

- un groupe musculaire profond, invaginateur.

La connaissance de ce système musculaire, observé dans son ensemble topographique et fonctionnel, grâce à la taille microscopique du scolex échinococcique, apporte sa contribution à l'étude comparèe du scolex des formes larvaires et adultes des Téniidés et précise, pour les échinocoques en particulier, le mécanisme spécial de l'invagination et de la dévagination du rostre et des ventouses.

\section{Bibliographie}

Coutelen (F.). - Essai de culture in vilto de scolex et d'hydatides échinococciques. Ann. Parasit. hum. et comp., 1927, V, p. 8.

- Recherches sur le système excréteur des hydatides échinocccciques. Ann. Parasit., hum. et comp., 1931, IX, p. 423.

Goldschmidt (R.). - Zur entwicklungsgeschichte der Echinococcusköpfchen. Zoologischen Yahrbüchern, 1900, XIII, p. 467.

LEjGKart (R.). - Die Blasenbandwürmer und ihre Entwicklung. Giessen, 1856.

Nianyn (B.). - Entwicklung der Echinococcus. Arch. f. Anat. Physiol. $u$. wissenschaft. méd., 1862, p. 612.

liasmussen (V.). - Bidrag til Kundskab om Echinococcernes Udvikling in Vidensk. Meddel. Naturhist. Foren., Kjöbenhavn, 1865.

Wagener (G. R.). - Die Entwicklung der Cestoden nach eigenen Untersuchungen, in Verh. Léop. Carol. Akad. Naturf., vol. XXIV, Suppl., BreslauBonn, 1854.

(Laboratoire de Parasitologie et de Pathologie parasitaire de la Faculté de Médecine de Lille) 
Figures illustrant le mémoire

de MM. F. Coutelen, J. Biguet, J.-M. Doby et St. Deblock 


\section{LEGENDE DE LA PLANCHE I}

Nota. - Les figures $1,2,3$ et 4 de cette planche I sont schématiques. Elles ont été dessinées, d'après nature, au moyen de préparations examinées à frais de scoiex echinococciques, les uns normaux et arrivés à un complet développement, les autres ayant subi, in vitro et à des degrés plus ou moins avancés, l'évolution vésiculaire régressive. Elles sont le résultat d'observations et de dessins partiels très nombreux. Chaque figure reconstitue synthétiquement, non pas toutes les fibres musculaires qu'il eût été impossible d'y inserire, mais avec, toutefois, leur densité relative, les grands groupes musculaires, leurs lignes de force et leurs insertions. Ce système musculaire compliqué explique la diversité et la complexité des mouvements que l'on peut enregistrer sur des scolex vivants que l'on fait sortir de leur quiescence par simple réchauffement, ou, mieux encore, par adjonction de bile au milieu dans lequel on les observe.

Fig. 1. - Vue optique d'ensemble des fibres musculaires sous-cuticulaires avec la couche de fibres musculaires la plus externe et, immédiatement sousjacente, la couche de fibres musculaires longitudinales. Pour la netteté du dessin, ie nombre de ces fibres a été réduit environ de moitié (voir sur le frais, la microphotographie $\mathrm{N}^{\circ} 5$ ).

Tis. 2. - Coupe optique antéro-postérieure passant un peu au-dessous de la zone sous-cuticulaire, entamant la partic antérieure du rostre, les crochets, la partie externe des deux ventouses situées dans le plan du schéma et, à la partie postérieure du scolex, la zone sous-cuticulaire du pédicule invaginé qui, en fait, est plus central.

Fıg. 3. - Coupe optique antéro-postérieure passant par l'axe de symétrie médian du scolex. Pour faciliter la compréhension du système musculaire rétracteur des ventouses, le dessin comporte aussi, dans sa partie supérieure, une coupe schématique qui les intéresserait dans leur partic moyenne et passerait en avant du grand axe antéro-postérieur, mais en arrière toutefois de la coupe optique de la figure 2.

Fic. 4. - Cette figure montre la projection des figures 1,2 et 3 sur un plan qui, situé en avant du rostre du scolex, serait perpendiculaire à son grand axe de symétrie antéro-postérieur. Les figures 1 et 3 occupent chacune un quartier de la projection (respectivement en haut et à gauche), la figure 2 l'autre moitié (à droite et en bas). Elle montre, sous un autre aspect, l'ensemble de ce système musculaire. 
ANNALES DE PARASITOLOGIE

T. XXVII, No 1-2-3, 1952.
Planche I

(Mémoire Coutelen et coll.)

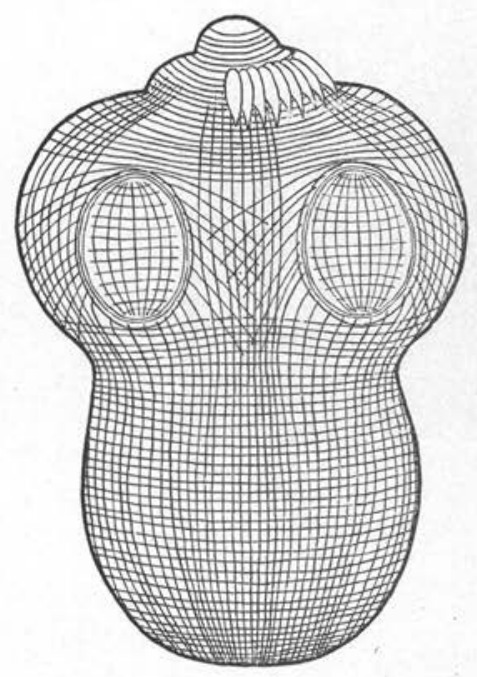

FIG. 1

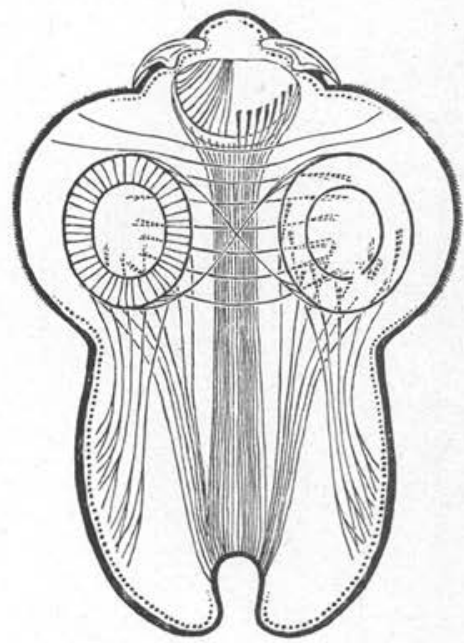

Fig. 3

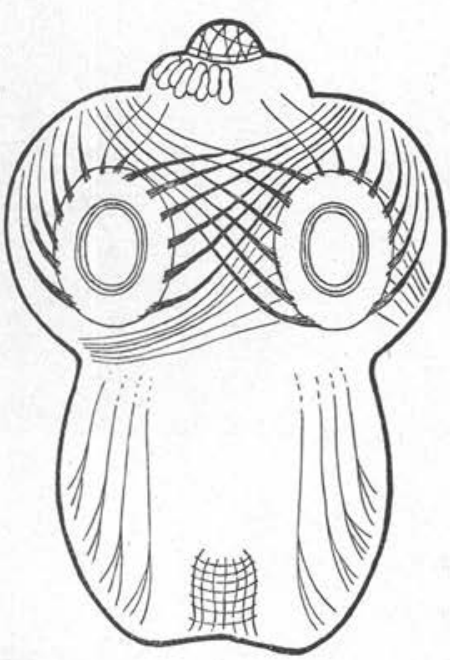

FIG, 2

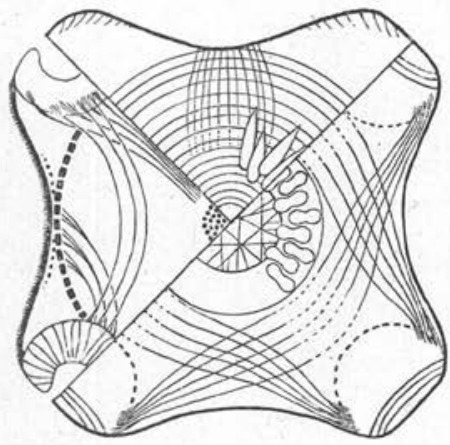

Fig. 4

Masson et $C^{i e}$, Editeurs 
Planche II

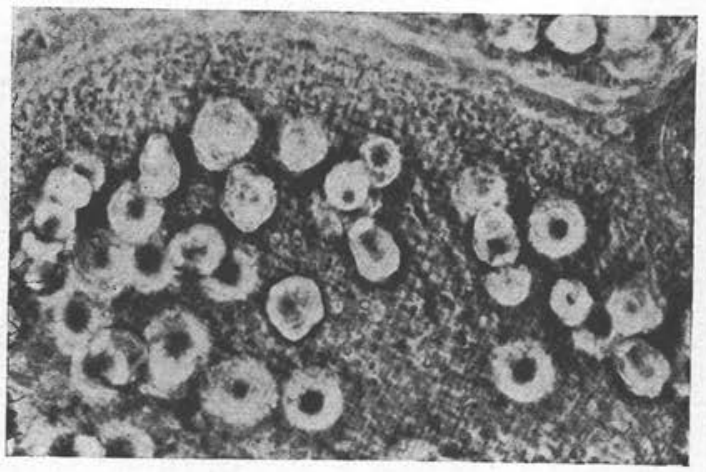

FIG. 5

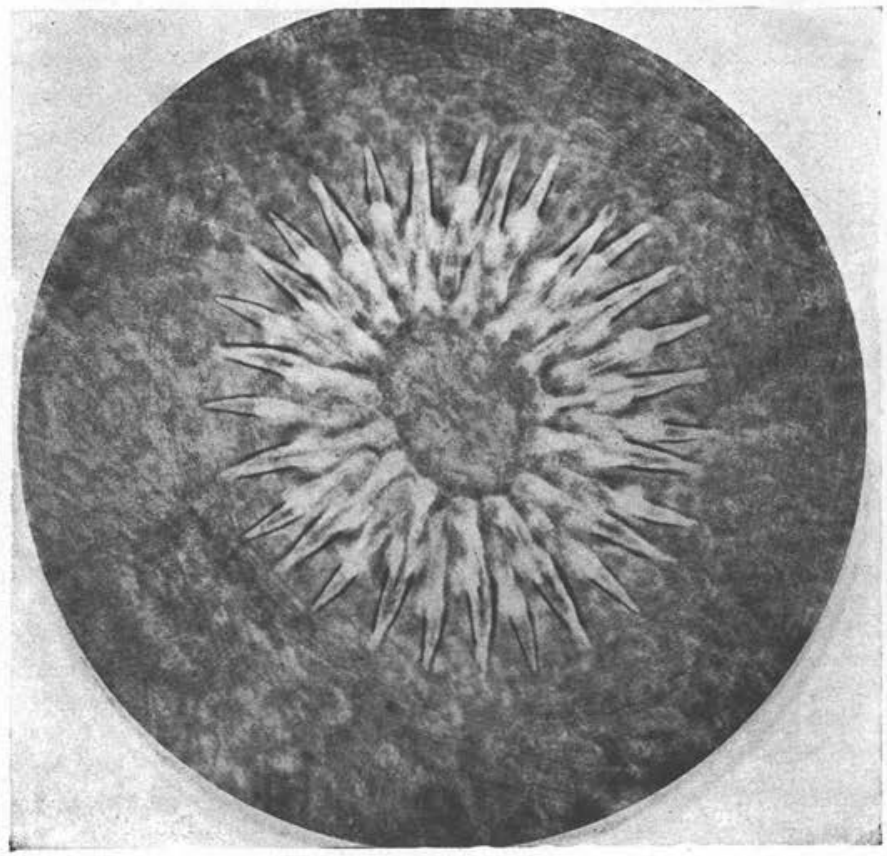

Fig. 6 


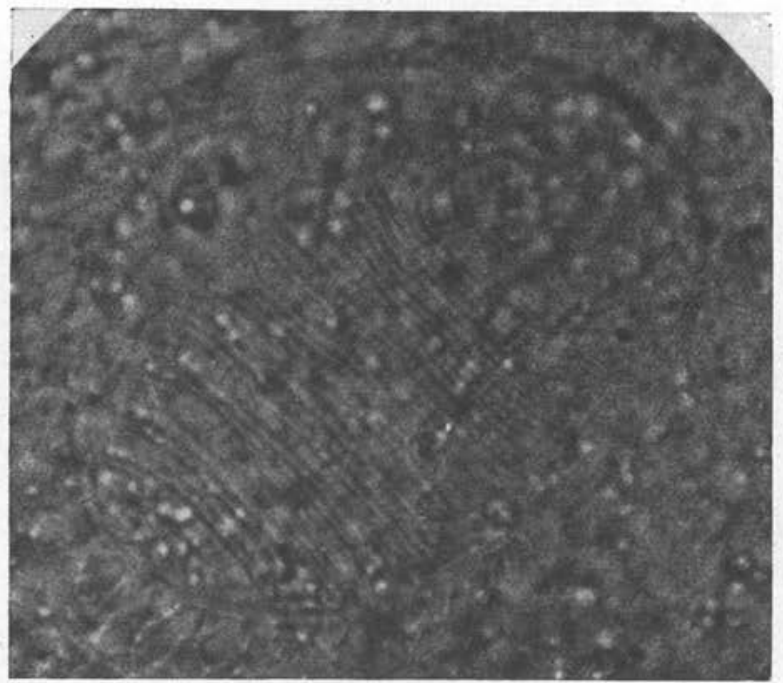

Frg. 7. - Microphotographie, à frais, d’un scolex à un stade avancé de vésiculation. Fond d'une ventouse. Quadrillage des fibres musculaires entrecroisées de la couche circulaire externe et de la couche longitudinale interne de la zone sous-cuticulaire. Quelques fibres circulaires périphériques.

\section{LEGENDE DE LA PLANCHE II}

FıG. 5. - Microphotographie, à frais, d'un scolex au début de la vésiculation. Partie postérieure. Zone sous-cuticulaire. Quadrillage serré, formé par la couche externe des fibres circulaires et, immédiatement au-dessous d'elle, par la couche des fibres musculaires longitudinales. Ces deux systèmes de fibres s'entrecroisent perpendiculairement. Noter l'extinetion optique de ces fibres au niveau des corpuscules calcaires réfringents et brillants.

Fig. 6. - Microphotographie, à frais, d'un scolex au début de la vésiculation. Zone sous-cuticulaire de la partie antérieure du rostre. Autour des extrémités des crochets et dans les parties les mieux venues, on distingue les fibres musculaires externes superficielles. 
Planche IV

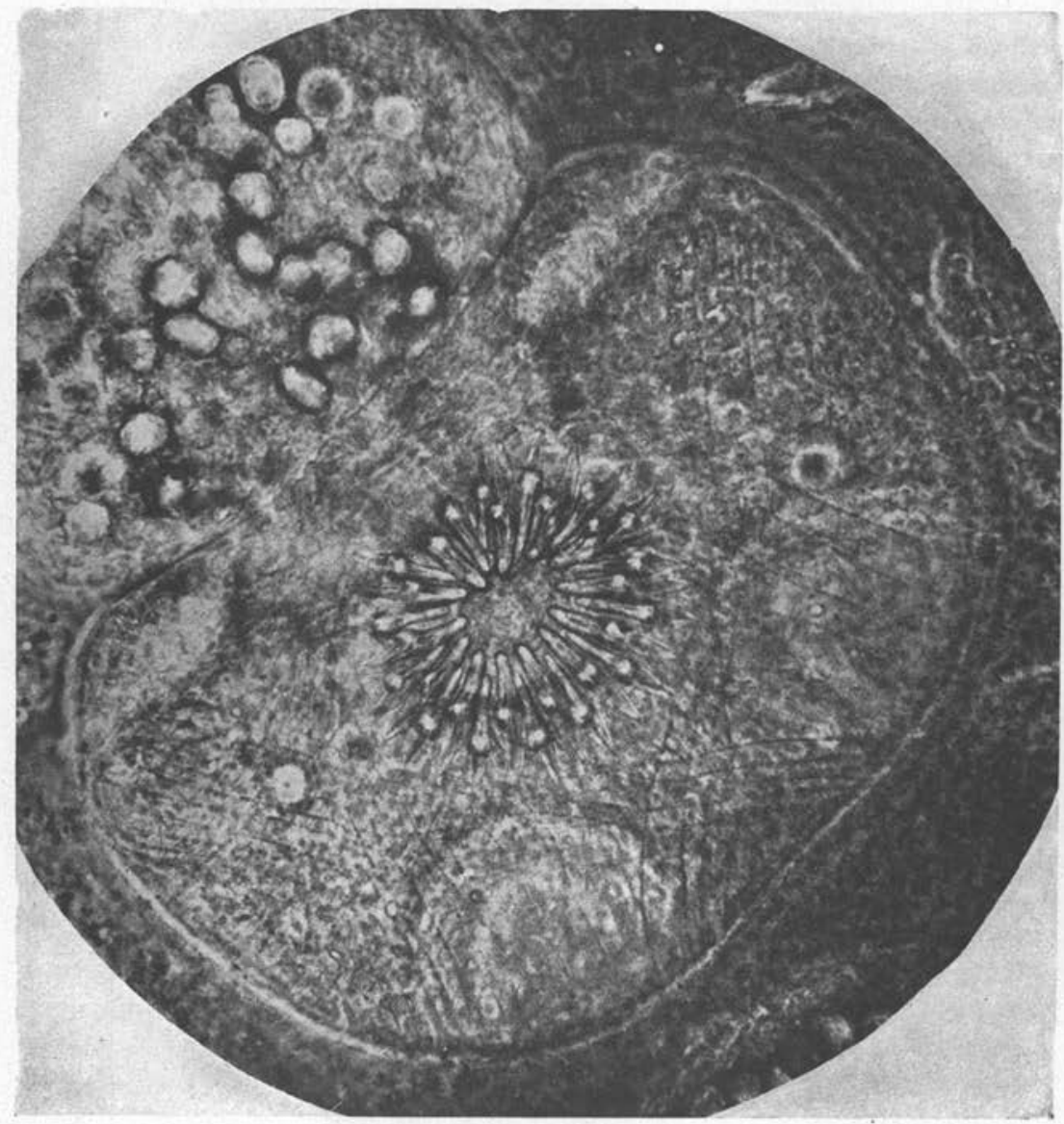

Fig. 8. - Microphotographie, à frais, d’un scolex contracté vu en partie par la face antérieure du rostre. Remarquer la netteté des chiasmas des grosses fibres musculaires insérées sur les ventouses, cans une zone plus profondément située que la sous-cuticule.

Cette microphoto, non plus que les autres, n'a fait l'objet d'aucune retouche. 


\section{Planche V}

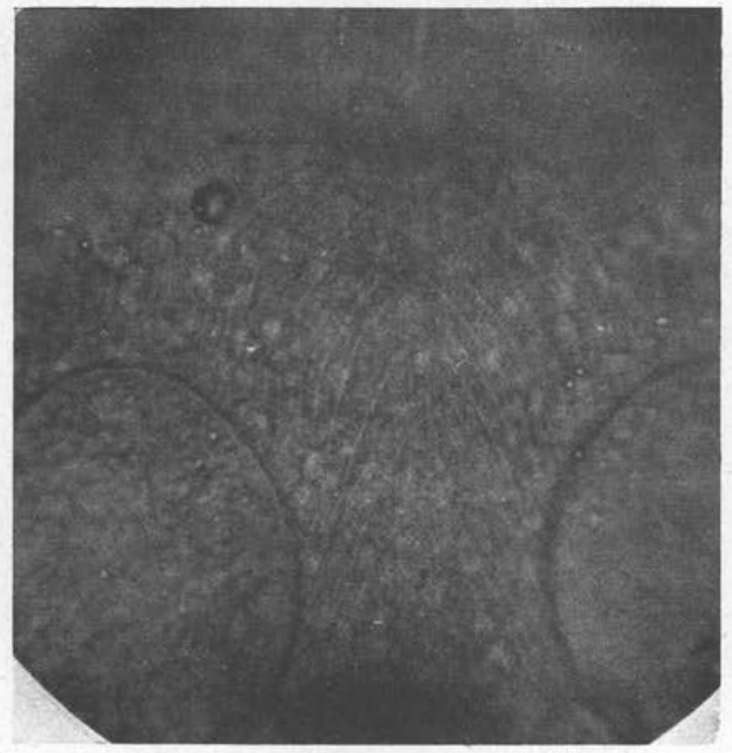

Fig. 9. -- Microphotographie, à frais, d'un scolex vésiculeux. Extrémité antérieure. En haut. l'extrémité de quelques crochets. Sur la ligne médiane, entre les deux ventouses et un peu au-dessous de la zone sous-cuticulaire dont on distingue encore la bande de fibres musculaires longitudinales internes, le chiasma des grosses fibres musculaires qui s'insèrent sur les ventouses et contournent la base du rostre du côté opposé. 


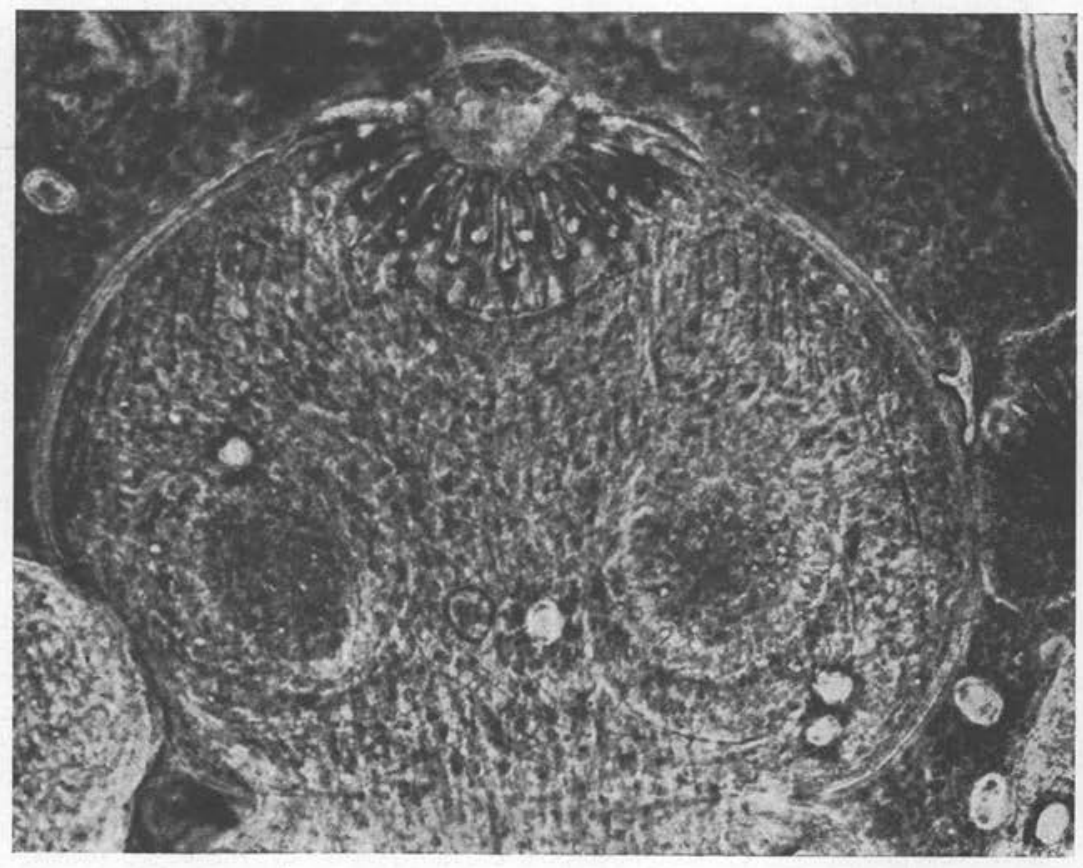

Fig. 10. - Microphotographie, à frais, de la partie antérieure, contractée, d'un scolex. Coupe optique passant par la face profonde des ventouses et la zone d'implantation des crochets. Noter, en particulier, autour de la base du rostre les fibres musculaires provenant du chiasma superficiel et, en arrière des ventouses, les fibres du chiasma profond dont on distingue encore l'entrecroisement sur la lignel médiane. 


\section{Planche VI}

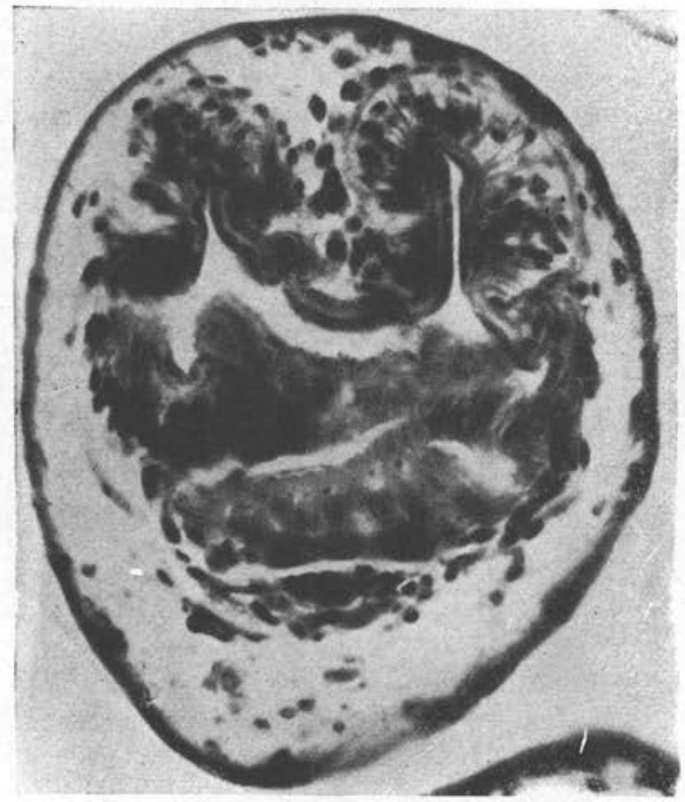

Fid. 11. - Microphotographie d'un scolex invaginé en voie de vésiculation. Coupe histologique transversale et oblique intéressant deux ventouses et la base du rostre. Pièce fixée au formol avec post-chromisation. Coloration de Benda. Cette présentation met en évidence les myoblastes et les fibres musculaires rayonnées des ventouses et également les fibres antéro-postérieures du bulbe rostral. 


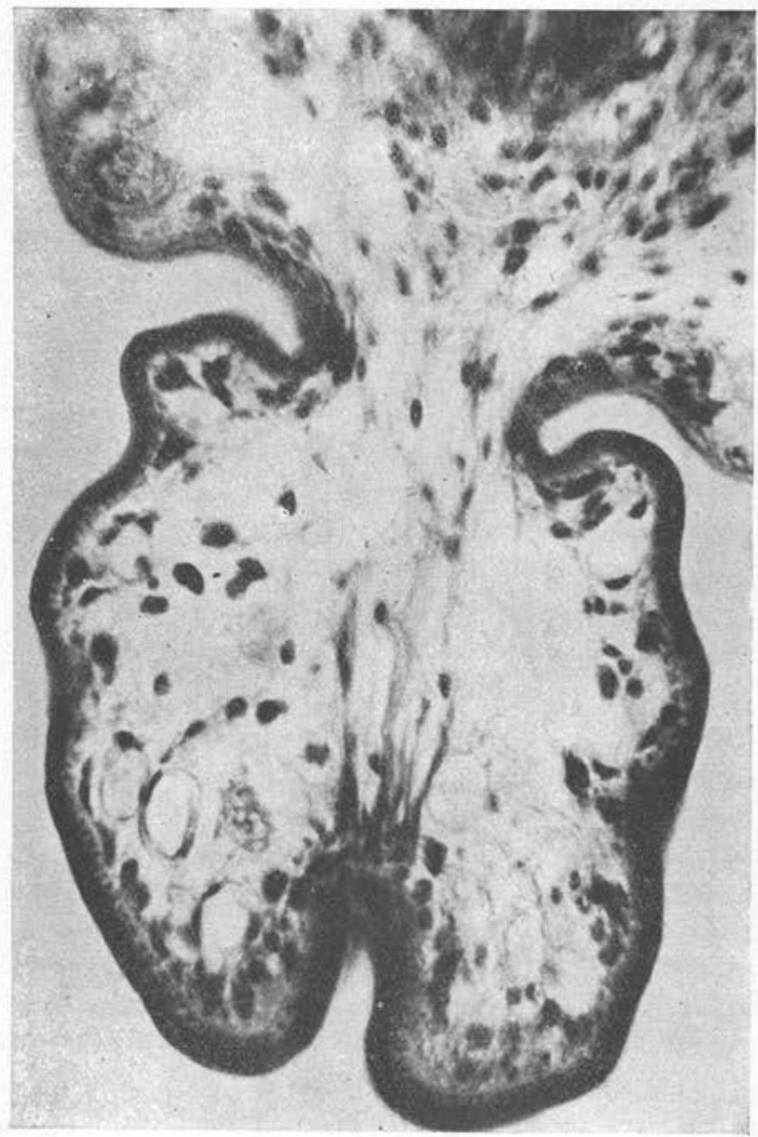

Fri. 12. - Microphotographie d’un scolex dévaginé en voie de vésiculation. Coupe histologique antéro-postérieure passant au voisinage du grand axe. Même fixation et mème coloration que dans la figure 11 .

On distingue, dans la zone sous-cuticulaire de la partie postérieure du scolex, les coupes transversales punctiformes des fibres musculaires circulaires externes et. sur le fond invaginé du pédicule, l'insertion du faisceau antéropostérieur de fibres museulaires centrales, invaginateur. 